\title{
World Wide Web Interface to Digital Imaging and Communication in Medicine-Capable Image Servers
}

\author{
Grover C. Browning, Yun Liang, Kenneth A. Buckwalter, Robert A. Kruger, and Alex Aisen
}

\begin{abstract}
As a trial project, the Indiana University Department of Radiology has developed a low-cost manner of distributing radiological images throughout a medical environment using the World Wide Web (WWW). The interface requires the user to have a WWW-browser client, such as Netscape, running on UNIX, PC, or Macintosh platforms. A forms-based interface allows the user to query several DICOM-capable machines at the machine, patient, study, series, and image levels. Once an image transfer is initiated, images are prewindowed from 16- to 8-bits, compressed using public domain Joint Photographic Expert Group (JPEG) compression routines, transferred to the WWW client program, and decompressed and displayed using a locally selected image viewing program. At the currently implemented level of compression $175 \%$ quality), the entire fetch-transform-JPEG-display process takes $\mathbf{2}$ to $\mathbf{5}$ seconds over Ethernet, depending on the platform used.
\end{abstract}

Copyright 1996 by W.B. Saunders Company

KEY WORDS: picture archiving and communication system (PACS), digital imaging and communication in medicine, World Wide Web, image display, image processing.

$\mathbf{P}$

ICTURE archiving and communication systems (PACS) ${ }^{1}$ generally consist of three portions: archiving, communicating, and displaying images. The display of radiological images often relies on expensive, high-resolution monitors that can display 1,500 to 2,000 lines of data. Although many people in a hospital environment (especially outside the Radiology Department) would like access to images stored on the archival system, few can afford the expensive display stations required.

As a trial project, the Indiana University Department of Radiology has developed a lowcost manner of distributing radiological images

From the Division of Imaging Science, Department of Radiology, Indiana University Medical Center, Indianapolis, $I N$.

Supponed in part by Heath and Human Senices grant U0I-HS08750.

Address reprint requests to Grover C. Browning, BA, Department of Radiology, Division of Imaging Science, Indiana University Medical Center, 541 Clinical Dr, Room 120. Indianapolis. IN 46202-5111.

Copwight c. 1996 by W.B. Saunders Company

0897-1889/96/0904-00104\$3.0010 throughout a medical environment using the World Wide Web (WWW). ${ }^{2}$ The interface requires the user to have a WWW-browser client, such as Mosaic or Netscape, running on UNIX, $\mathrm{PC}$, or Macintosh platforms. The WWW functions as a distributed client/server system in which low-cost clients, running on a variety of platforms, can be used as a generic interface to information of all kinds including radiological images. Because the intended use of this image review capability is not primary diagnosis, lossy image compression was incorporated in the design to speed transfer over local area networks or via modem.

The Indiana University Department of Radiology provides services to five hospitals: Richard L. Roudebush Veterans Administration Medical Center, Wishard Memorial Hospital, James Whitcomb Riley Hospital for Children, University Hospital, and Johnson County Memorial Hospital. Distributed throughout these hospitals are six digital imaging and communication in medicine (DICOM)-capable computer tomography (CT) machines and five DICOMcapable magnetic resonance imaging (MRI) machines. DICOM ${ }^{3}$ is a communication protocol used in the medical imaging community. Its chief use is the transmission of medical image data between two computers. DICOM runs on top of the TCP/IP communication protocol. These 11 devices feed their image data, through DICOM, into a 7-unit distributed PACS system. Image data from any one of the hospitals are available through any orie of the seven PACS archives.

\section{WWW/DICOM GATEWAY}

The WWW/DICOM gateway uses a standard $\mathrm{CGI}^{4}$ script program. When a WWW client program, such as Mosaic or Netscape, connects to the WWW image server to request image information, a CGI program is spawned on the image server to handle the request. This CGI program takes the data provided from the WWW server and makes a DICOM request of a DICOM host. The information requested, either an image or an HTTP form, is then 
returned to the WWW server. The WWW server then sends the information to the WWW client that originated the procedure.

The WWW/DICOM gateway can generate five types of data to be returned to a user. It can present a list of machines that can be queried, a list of studies on one of those machines, a list of series in one of those studies, a list of images in one of those series, or the actual image. Four of these instances involve the return of an HTTPD image form, whereas the fifth returns image data. Three of the forms return data found by executing a DICOM query of a DICOMcapable machine. The fourth, a list of DICOMcapable machines, is generated from a configuration file.

\section{Machine-Level Query}

Figure 1 shows the first screen a user sees when connecting to http://www.indyrad.i- upui.edu/cgi-bin/wwwdcmnew. This is the image device list. This screen presents all of the devices capable of sending images, as well as three delimiters for any potential search of the machines. The users can constrain a search by specifying a patient name, a patient identifier (ID), or a date range. The list of machines is represented by a selection box, with the form tag of MACHINE. The selections within this list box are read from a configuration file.

To find specific patients in the PACS, two edit boxes are presented to allow constrained searches. The first of these is the patient name edit box, with the form tag NAME. This edit box allows three types of input. The first is a specific patient name, such as "Browning, Grover." In this case, the DICOM query of the DICOM machine selected will return only the patient with the specified name. The second type of query is a wild-card query, such as "Bro*in?"
Fig 1. A screen capture showing the machine-level user screen.

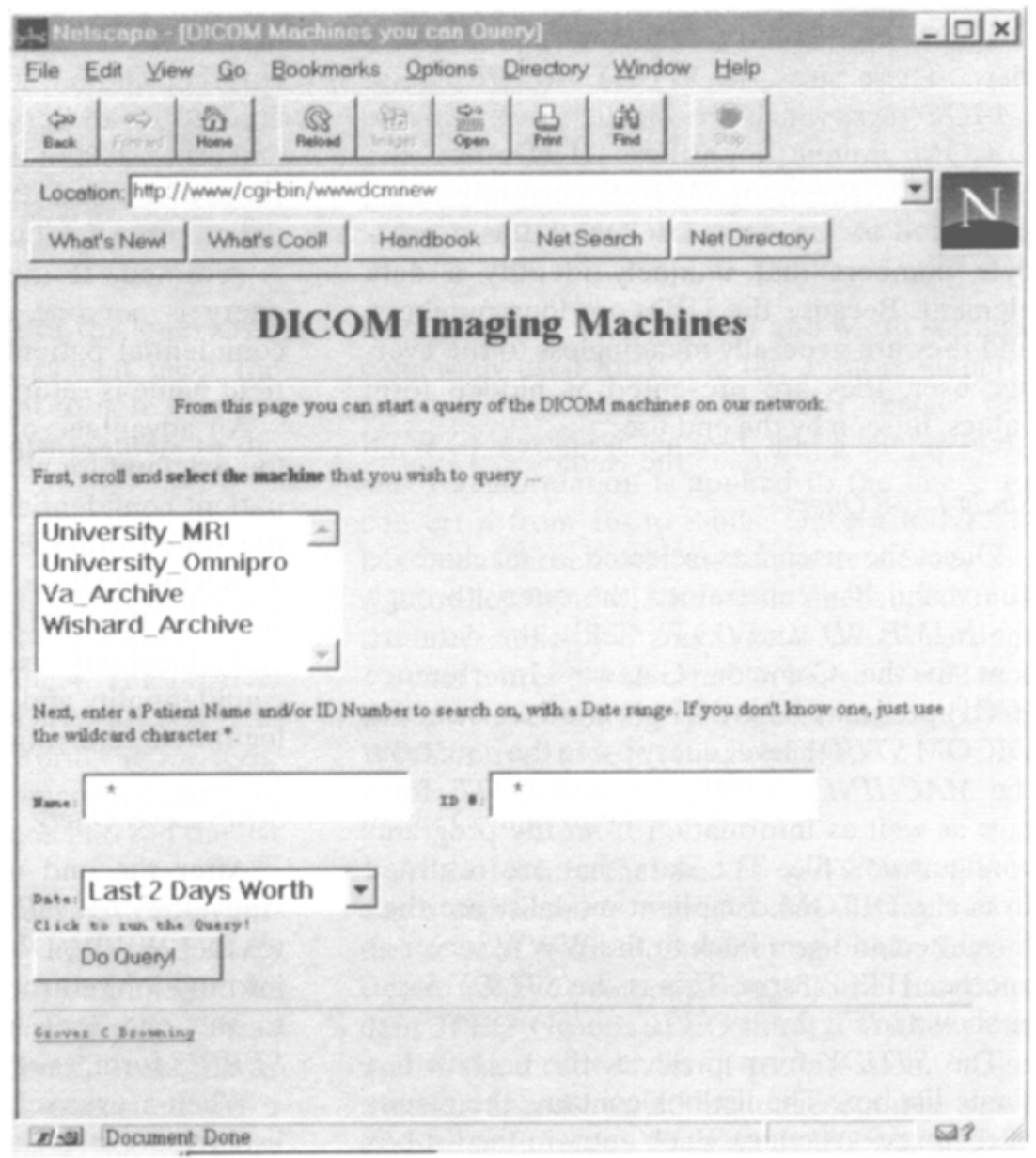


This allows for any number of characters to be substituted for "** and any single character to be substituted for the "?" The third type of query is a generalized version of the wild-card query, a universal query, employs a single character "*" to indicate that all names should be returned.

The patient ID edit box with the form tag $I D$ behaves in the same way as the patient name edit box, allowing searches on specific, wildcard, or universal values.

The third item that can be used to constrain the DICOM query of the machine selected is a date range. Four values are presented to a user by means of a selection box. Users can search for study data generated in the last 2 days, the last week, the last month, or the user can request that all data, regardless of the date generated, be returned. The date range form tag is the value $D A T E$, and the default value is for all data to be returned.

Three other form tags that are present on the machine-level screen are hidden from the user's view. These are the STUDY, SERIES, and $I M A G E$ tags, which are meant to contain the DICOM unique identifiers (UIDs) of the STUDY, SERIES, or IMAGE currently being presented to the user. DICOM UIDs are 32byte numbers that uniquely identify a data element. Because the UIDs are long numbers, and they are generally meaningless to the average user, they are presented as hidden form values, unseen by the end user.

\section{Study-Level Query}

Once the user has selected a machine to query and has constrained the query through the $N A M E, I D$, and DATE fields, the data are sent to the Common Gateway Interference (CGI) program. The CGI program constructs a DICOM STUDY level query using the data from the MACHINE, NAME, ID, and DATE form tags as well as information from the programs configuration file. The data that are returned from the DICOM compliant modality are then formatted and sent back to the WWW server as another HTTP form. This is the STUDY form, as shown in Fig 2.

The STUDY form presents the user with a single list box. The list box contains the results of the previous query. Each entry in the list box contains the name of the patient, the study date, and a short description. This list box is associated with the STUDY form tag. Each entry in the list box has an associated DICOM Study UID. After an entry from the list box is chosen, the STUDY form tag is sent to the DICOM Study UID of the chosen patient study.

The STUDY-level user screen has two other important associated processes. The first is a hidden form tag, the MACHINE tag. This hidden tag contains the value of the machine chosen on the machine-level screen. This indicates to the CGI program which DICOM machine should be consulted for the next query that the user requests.

The second important process deals with patient confidentiality. The security scheme is based on the Internet Protocol (IP) address of the user requesting the query. As a part of the general HTTP forms interface, the IP address of the user of the WWW client program is sent to the WWW/DICOM gateway. The configuration file for the CGI program contains space for entering allowed IP addresses and ranges of allowed IP addresses. If the IP address of the end user is one of those addresses or falls in the range of allowed IP addresses, then confidential patient information, such as the patients name, is returned. If the end user performing the query is not one of the few allowed to see confidential patient information, then the patient name is returned as "Name is restricted."

An advantage of this scheme is that the de facto default for all IP addresses limits access to patient confidential information, unless explicitly stated otherwise in the configuration file. This feature allows a causal user to acquire data from the WWW/DICOM gateway for research and educational uses, but still preserves patient confidentiality and the access to the data that a legitimate referring physician may need.

\section{Series-Level Query}

After the end user has selected a patient study, the MACHINE and STUDY tags are sent to the WWW/DICOM gateway. The series information returned is formatted and then sent to the end user as another httpd form, the SERIES form, as shown in Fig 3.

When a user selects a series from the series list box several form tags are sent to the WWW/ 
Fig 2. A screen capture showing the study-level user screen. Patient names have been blacked out to preserve patient confidentiality.

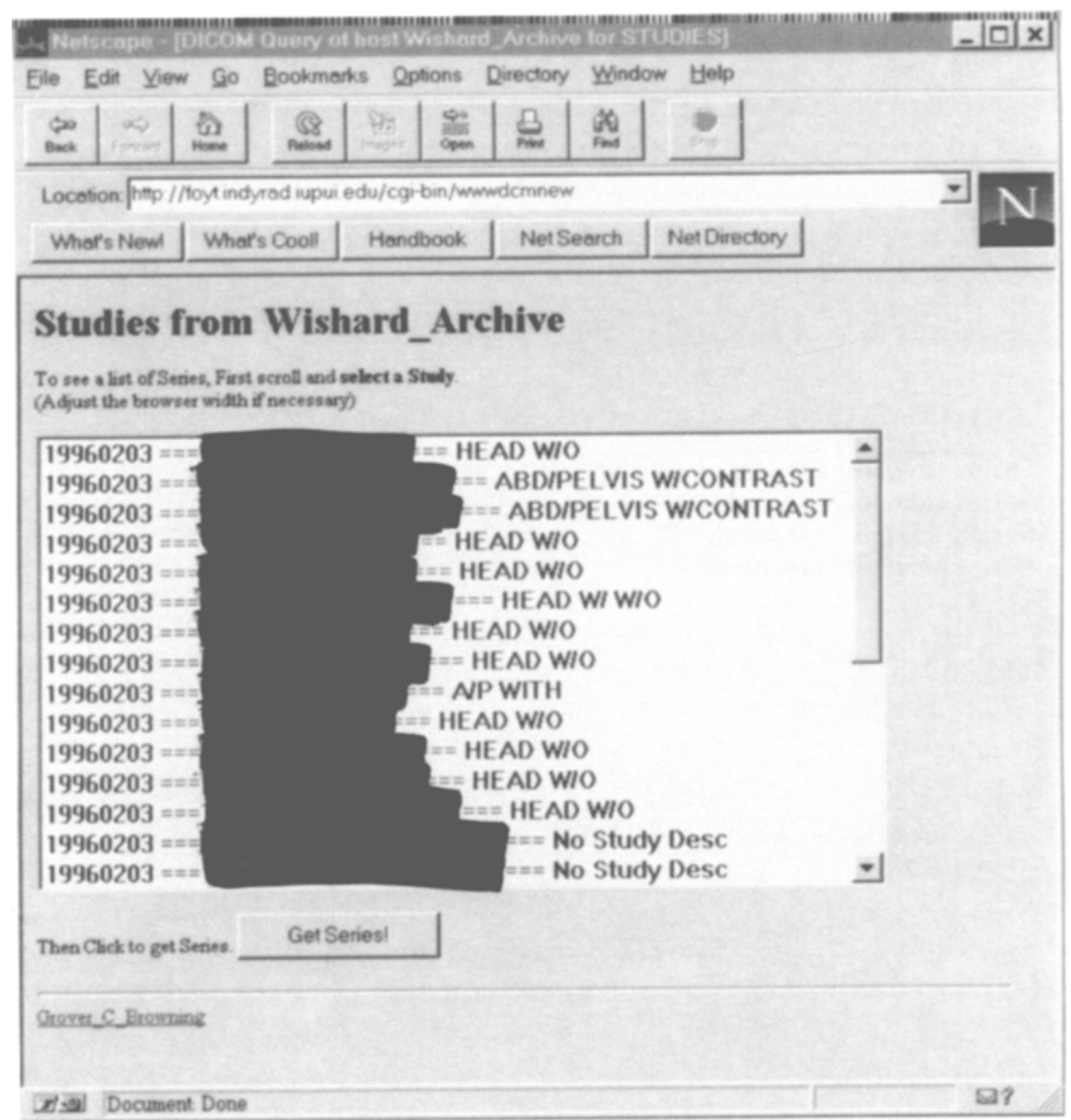

DICOM gateway. The result of this query is a reference to all of the images that meet the search criteria. It is important to note that the actual images are not sent, just pointers to the images.

\section{Image-Level Query}

The data returned by the DICOM query are formatted and returned as another httpd form, the IMAGE form, shown in Fig 4. The IMAGE form contains a list box and a drop-list box. The list box, associated with the form tag IMAGE, contains one entry for each image available in the series. Associated with each entry in the list box is a DICOM Image UID.

The drop-list box contains several preset image display settings (window width and level) that are used to transform the 16-bit image data to an 8-bit representation for display. These settings are labeled Bone1, Bone2, Spine, Lung, and Abdomen. This drop list is associated with a WINLEV form tag. These settings duplicate the most common window level and width settings commonly used for CT in the authors' department. If the image is an MRI image, the WINLEV setting is ignored, and a simple min/ max transformation is applied to the image to convert it from 16- to 8-bits. Once a WINLEV has been chosen and an image retrieved, the window level and width remain unchanged until the user explicitly changes the WINLEV setting.

After a user has selected an image as well as a window/level setting, a request made to the WWW server to retrieve the image.

\section{Image Compression}

A key feature that makes our WWW/Gateway a viable query, retrieval and display tool is the use of 8-bit Joint Photographic Expert Group (JPEG) compression, via the Independent JPEG Groups JPEG library. Without the use of significant levels of compression, image transfer speeds would be unacceptably slow, even over Ethernet. Eight-bit JPEG was chosen 


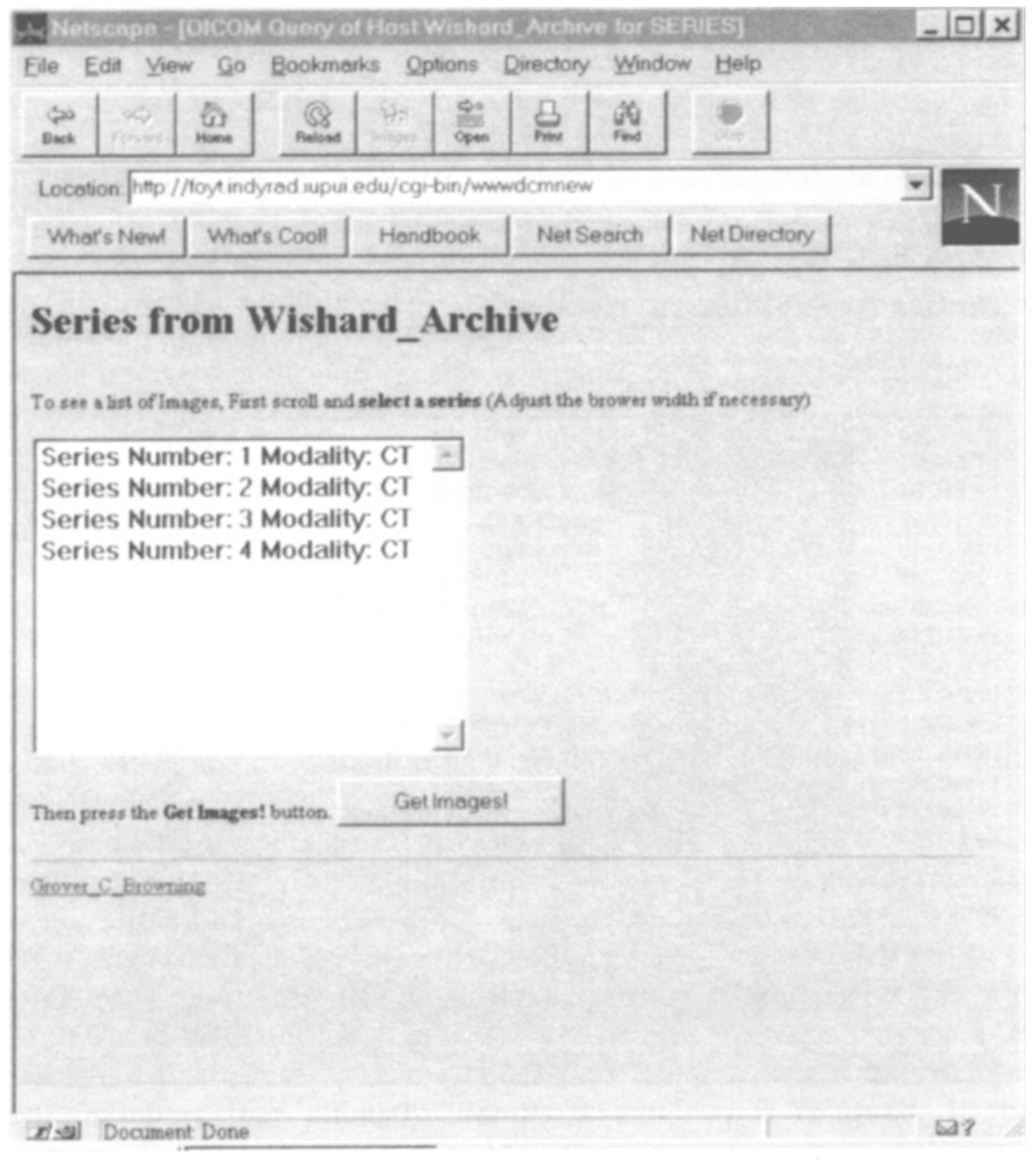

Fig 3. A screen capture show. ing the series-level user screen.

as the authors' compression algorithm because it resides in the public domain and can be decoded with a variety of image display software including those resident in WWW browser clients, such as Mosaic or Netscape. Although the use of 16-bit JPEG would have allowed for retrospective window-leveling control, 16-bit JPEG is not commonly available to the causal user, and few display programs can handle 16-bit, gray-scale data. All things considered, the authors thought it preferable to perform windowing through the WWW/DICOM gateway as described in the Image-Level Query section and to deal with 8-bit data thereafter. An example of one of these images is shown in Fig 5.

JPEG $^{5}$ defines a family of lossy compression algorithms based on the discrete cosine transform. The degree of JPEG compression is chosen indirectly by selection of a quality factor, which ranges from $0 \%$ to $100 \%$. The lower the quality factor, the greater the amount of com- pression. As currently implemented, we are using an empirically determined quality factor of $75 \%$. At this level of compression, a "typical" CT image $(512 \times 512 \times 16)$ is compressed from 0.52 Mbytes to $20-25$ kbytes.

\section{Configuration File}

Most of the configuration of the WWW/ DICOM gateway program comes from a simple text configuration file. Every time a new request is made of the gateway program, the configuration file is consulted. Thus, changes to the configuration of the gateway program take effect almost immediately.

The configuration file itself is made up of three general areas: $W W W$ configuration, the patient security information, and DICOM configuration. The entire file is arranged in a $\langle$ tag (value) format. The WWW/DICOM gateway searches the configuration file for a specified 〈tag) and selects the 〈value〉 associated with that $\langle$ tag $\rangle$. 
Fig 4. A screen capture showing the image-level user screen.

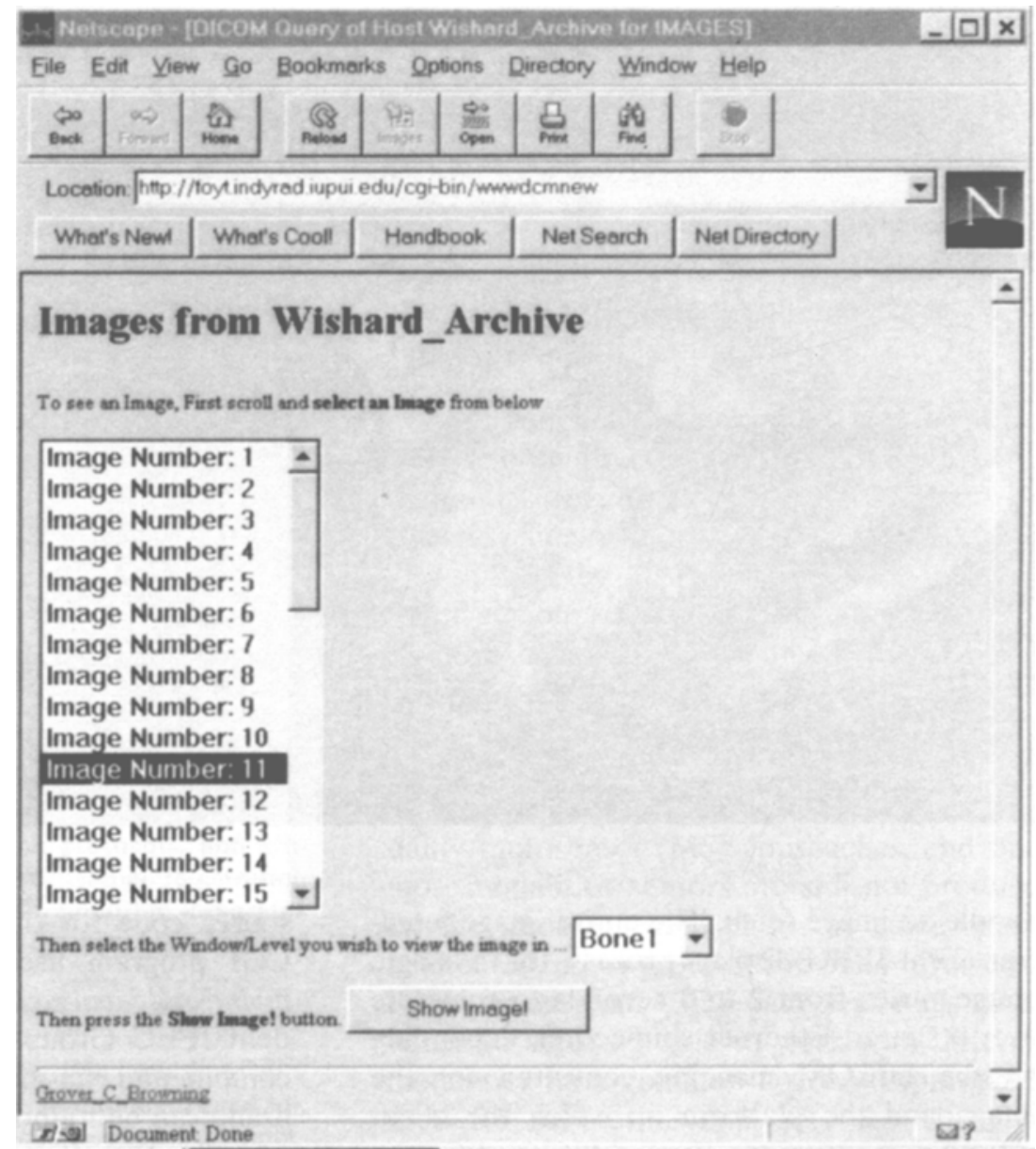

The $W W W$ configuration file deals with the WWW portions of the gateway program. As such, it contains 〈tag v values representing the address of the WWW server, and $\langle$ value $\rangle$ entries specifying the icons or logos that appear on the gateways HTTP forms, and contact information if a user has questions about the data retrieved.

The patient security file consists of multiple $\langle\mathrm{tag}\rangle\langle$ value $\rangle$ pairs, the $\langle$ tags $\rangle$ of which are of one form, ALLOW. Each ALLOW tag is followed by either a unique IP address, or an A-, B-, or $C$-class IP address. Any user of the system that has an IP address included in an $A L L O W$ value has the ability to see information, such as the patient name that is denied to other users.

The DICOM configuration consists of 〈tag) (value) pairs, the first of which is the MYAE tag. The value associated with this tag is the DICOM Application Entity (AE) title of the computer running the WWW/DICOM gateway. A DICOM AE label is used in DICOM communications to identify a DICOM machine in the local environment uniquely. The $\mathrm{AE}$ value is required by the DICOM standard to be included in all DICOM communication. The second 〈tag) 〈value〉 pair describes the DICOM information associated with the equipment to be queried for images. These entries are denoted by the MACHINE tag. Each MACHINE tag in the file has four data elements making up the 〈value portion of the $\langle$ tag $\rangle$ value pair: IP address or IP hostname of the DICOM equipment, the DICOM AE of the DICOM device, the common name of the DICOM machine being consulted, and the TCP port number through which the DICOM device expects all DICOM information to arrive.

\section{PERFORMANCE}

Dedicated PACS equipment tends to respond quickly to image requests, whereas modality-specific equipment tends to take longer to 


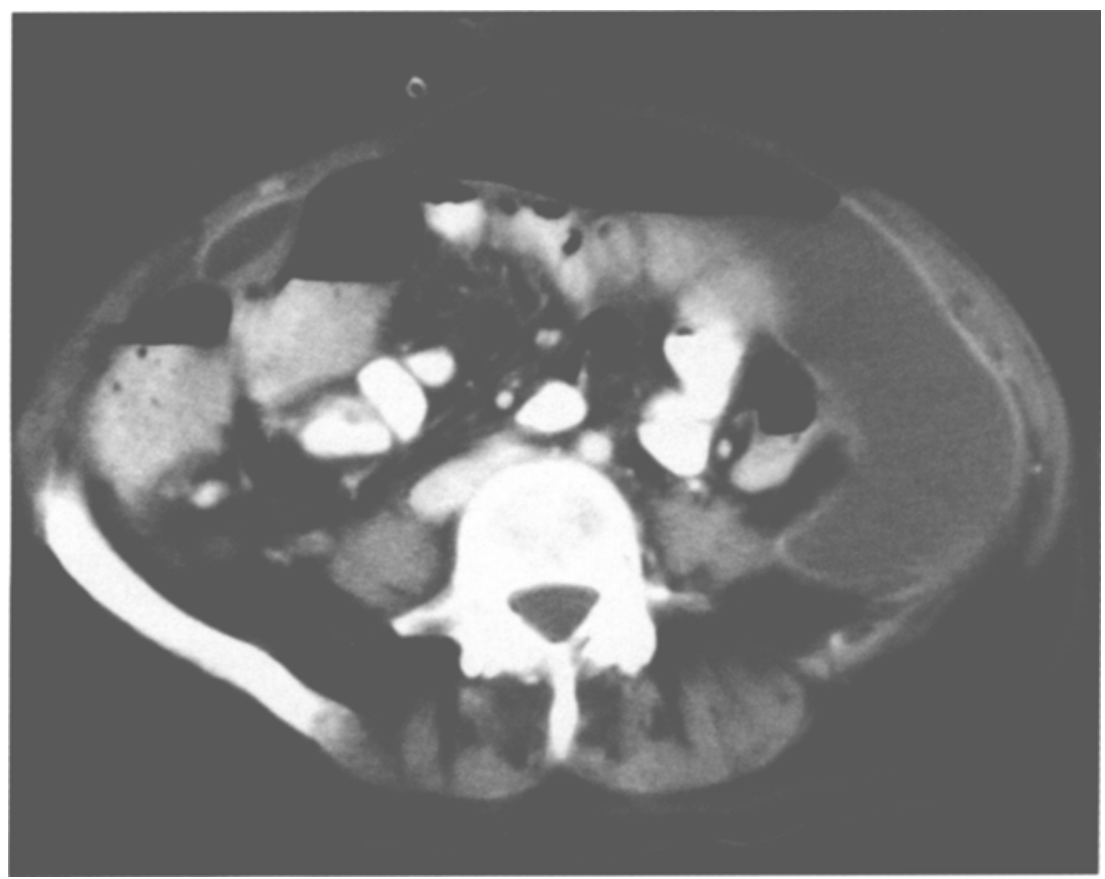

Fig 5. A screen capture show. ing a JPEG image of abdominal image that has been windowed and leveled. handle an image fetch. The entire image fetchtransform-JPEG-display process for a single image varies from 2 to 5 seconds to complete over a shared Ethernet connection, depending on the DICOM machine consulted for the image and the platform on which the client software is running. For a $486 / 66 \mathrm{PC}$, the process for a CT image takes about 5 seconds and 3 seconds for an MRI $(256 \times 256)$. Over a 14.4-kbaud modem, the process takes about a factor of $\times 4$ longer.

\section{SOFTWARE AVAILABILITY}

The source code for the CGI program that acts as a WWW/DICOM gateway, as well as the source code for the DICOM library that the CGl program uses, can be found at $\mathrm{ftp}: / /$ fip.indyradiupui.edu/pub/DICOM. The Independent JPEG Groups JPEG library, required to compile and run the CGI WWW/DICOM program, can be found at ftp://ftp.uu.net/graphics/ jpeg. The WWW server used for this application is the Netscape Communications Enterprise Server 2.0. It, as well as the WWW/DICOM CGI program, is running on a Hewlett Packard 9000/755 UNIX computer (Hewlett Packard, Palo Alto, CA). Any CGI compliant WWW server running on any UNIX computer platform should be able to support the WWW/DICOM CGI program.

\section{REFERENCES}

1. Cawkell AE: A Guide to Image Processing and Picture Management. Brookfield, VT, Gower, 1994

2. Castro, E: HTML for the World Wide Web. Berkeley, CA. Peachpit Press, 1996

3. National Electrical Manufacturers Association: Digital Imaging and Communications in Medicine (DICOM), United States, 1994. I. Introduction and Overview. Washing- ton, DC, National Electrical Manufacturers Association, 1994

4. Brenner S: Introduction to CGI/PERL: Getting Started with Web Scripts. New York, NY, M\&T Books, 1996

5. Pennebaker WB. Mitchell JL: JPEG Still Image Data Compression Standard. New York, NY, Van Nostrand Rainhold. 1993 